\title{
MR Diffusion-Based Inference of a Fiber Bundle Model from a Population of Subjects
}

\author{
V. El Kouby ${ }^{1,3}$, Y. Cointepas ${ }^{1,3}$, C. Poupon $^{1,3}$, D. Rivière ${ }^{1,3}$, N. Golestani ${ }^{1,2}$, \\ J.-B. Poline ${ }^{4}$, D. Le Bihan ${ }^{1,3}$, and J.-F. Mangin ${ }^{1,3}$ \\ 1 Service Hospitalier Frédéric Joliot, CEA, 91401 Orsay, France \\ elkouby@shfj.cea.fr, http: / / brainvisa.info \\ 2 INSERM U562 Cognitive Neuro Imaging, Orsay, France \\ 3 Institut Fédératif de Recherche 49 (Imagerie Neurofonctionnelle), Paris
}

\begin{abstract}
This paper proposes a method to infer a high level model of the white matter organization from a population of subjects using MR diffusion imaging. This method takes as input for each subject a set of trajectories stemming from any tracking algorithm. Then the inference results from two nested clustering stages. The first clustering converts each individual set of trajectories into a set of bundles supposed to represent large white matter pathways. The second clustering matches these bundles across subjects in order to provide a list of candidates for the bundle model. The method is applied on a population of eleven subjects and leads to the inference of 17 such candidates.
\end{abstract}

\section{Introduction}

In the recent past, the development of diffusion tensor imaging (DTI) has led to a new field of opportunities for the study of brain white matter [11]. A possible approach for such studies lies in the usual spatial normalization framework. For this purpose, the development of methods for warping tensor fields using full tensor information is a promising undertaking, because it may improve the normalization computed from standard anatomical images [10,20]. Spatial normalization can be used for instance for voxel-based statistical analysis. Combining normalization with fiber tracking techniques can also lead to statistical parametric maps reflecting variability of bundle location and thickness. Other morphometric approaches can be developped from dedicated parameterizations of the bundles [8,6].

One of the difficulties for bundle morphometry stems from the poor anatomical knowledge about white matter organization of the human brain. Most of the anatomical techniques used to trace tracts can not be used in the human brain. Therefore, the current knowledge built from Klinger method or observation of Wallerian degeneration after lesion is relatively sparse [7, 13]. There is a need for computational methods aiming at infering a model of the main bundles making up the human brain white matter from MR diffusion imaging. This paper explores this direction of research.

A lot of methods have been proposed to compute fiber related pathways from diffusion-weighted MR images. The simplest approaches follow trajectories of maximum diffusion [14, 5, 1]. More sophisticated methods try to overcome partial volume problems induced by fiber crossing using either high angular resolution acquisitions [18, 15], 
regularization [19, 16], front propagation [12,9] or Monte Carlo sampling [3, 2]. Most of these techniques convert the raw MR diffusion data into a huge set of trajectories supposed to correspond to putative fascicles. These fascicles can be used to create connectivity matrices related either to functional or anatomical regions of interest (ROIs) [5]. These fascicles can also be organized into larger bundles using clustering algorithms [4], which is addressed in this paper. This last approach is interesting because the potential influence of erroneous definitions of the ROIs is discarded.

The proposed method aims at the inference of a model of the bundles that can be detected in most of the subjects. Some of these bundles should be related to the current anatomical knowledge, but the ultimate goal is to enrich the current understanding of white matter organization. The method is made up of two nested clustering algorithms. The first clustering is performed subject by subject in order to reduce the complexity of the full set of fascicles to a small set of large bundles (cf. Fig. 1). The second clustering is performed across subjects in order to match similar bundles that may correspond to a generic anatomical pathway (cf. Fig.3 and 4). This second stage relies on a simple affine spatial normalization computed from the diffusion-free T2-weighted image included in the dataset. The work described in this paper is exploratory. Therefore, we do not address the current weaknesses of the tracking algorithms that may provide spurious fascicles. Moreover, we use a very simple clustering algorithm in order to get a first insight into the problem before designing more sophisticated dedicated methods.

\section{Method}

\subsection{Clustering the Fascicles}

The first clustering is performed subject by subject according to the following scheme:

Global fascicle tracking. The computation of the initial set of fascicles is performed with brainVISA (http://brainvisa.info), an open software implementing Mori's algorithm [14]. Simply speaking, the fascicles are trajectories of highest diffusion reconstructed step by step from the diffusion tensor field. A mask of the brain is computed from the T2-weighted image and one trajectory is obtained for each voxel, leading to a set of about 500000 fascicles. In this paper, we have chosen to focus on long bundles which led us to discard the fascicles whose length is less that $5 \mathrm{~cm}$. The final set includes about 20000 fascicles (see Fig,1]A).

ROI map. The clustering is not performed directly on the fascicles but on a set of Regions of Interest (ROIs) defined from Talairach proportional system. The Talairach grid is split into cubic ROIs that are transformed to the subject referential using affine spatial normalization of the T2-weighted image performed via SPM2 (http://www.fil.ion.ucl.ac.uk/spm) (see Fig 1,B). For the study described in this paper, the spatial resolution of the grid of ROIs is $5 \mathrm{~mm}$. After masking this grid with a mask of the subject white matter (see Fig 1D), the final set is made up of about 5000 ROIs.

Connectivity matrix. An anatomical connectivity matrix $A$ is computed for the set of ROIs mentioned above. For each pair of ROIs $(i, j), A_{i j}$ is the number of fascicles 


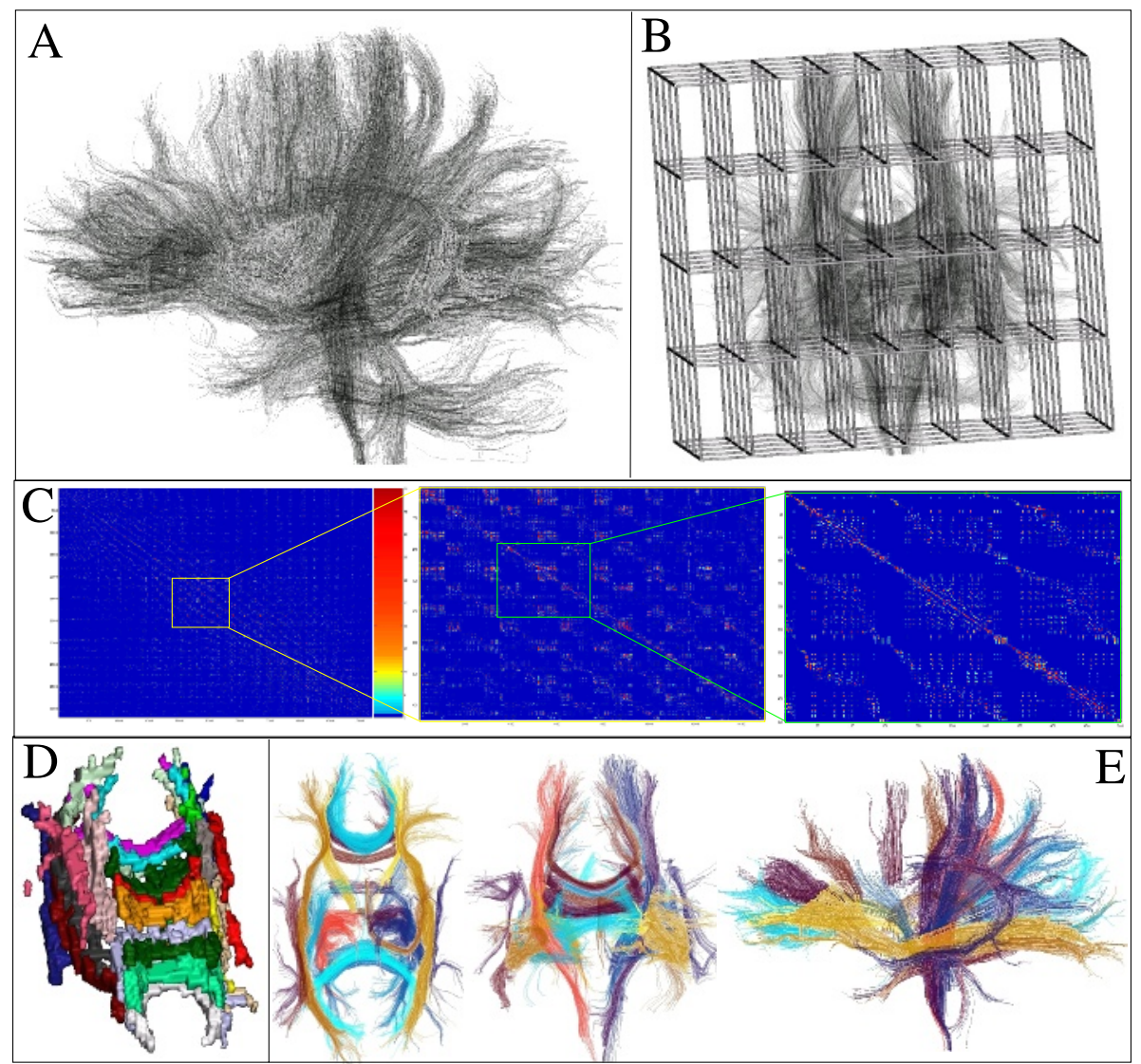

Fig. 1. Clustering the fascicles of one subject: A: The set of long fascicles to be clustered into bundles. B: The same set of fascicles embedded into the Talairach grid used to define cubic ROIs of $5 \mathrm{~mm}$ resolution. C: The connectivity matrix of the ROIs with 2 levels of zoom (about 5000 ROIs). Each point in the matrix is the number of fascicles linking the two related ROIs. D: Result of the clustering for the ROIs. Each color denotes a cluster. Each ROI shape is a cube in Talairach space masked by the subject white matter. E: Result of the clustering at the level of the fascicles. The garbage clusters have been filtered out.

crossing $i$ and $j$ (see Fig 1,C). In order to focus the clustering on the strongest connectivity information, this matrix is binarized using a threshold on the number of fascicles. For the experiment described further, this threshold has been set to the mean of non null coefficients plus one standard deviation, namely about 40 fascicles depending on the subject.

Clustering. In the matrix $A$, each row stands for the connectivity of one ROI with the other ROIs. The ROIs belonging to the same bundle should have similar connectivity patterns. Therefore, we perform a clustering among the ROI set using a distance between ROI corresponding to the Euclidean distance betwwen the rows of $A$. The 
clustering is performed using the standard k-means with random initialization of the centers (see Fig 1,D). K-means algorithm assigns each ROI to a cluster, which lead us to assume that the largest cluster in a kind of garbage collector that is discarded before further processing.

Fascicle bundles. The final step of the clustering consists in moving back to the fascicle world. Each cluster of ROIs is used to extract a subset of fascicles from the global set. For robustness purpose, a fascicle only needs to be partly included in the ROI cluster to be selected. In practice, a fascicle is selected when at least $60 \%$ of its sampling is included (see Fig 1]E). A last processing aims at splitting the resulting set of fascicles into connected components, which is required to deal with some unpredictable behaviour of the K-means. The clustering, indeed, gathers sometimes two bundles symmetric across interhemispheric plane, which calls for the use of better algorithms in the future. This postprocessing can not be performed directly at the level of the ROI clusters because the ROIs including fiber crossing can be assigned to only one cluster, leading sometimes to disconnect a cluster of ROIs corresponding to a reasonable bundle. The analysis of the connectedness of a fascicle set is performed via embedding of the fascicles into a $3 \mathrm{D}$ volume. It should be noted that this postprocessing implies that the final number of fascicle bundles depends on the subject.

\subsection{Clustering the Bundles Across Subjects}

The goal of the second stage of clustering is the inference of a model of the bundles that should be found in any human brain. The underlying idea is that a simple clustering performed to match similar bundles could provide a list of candidates for such a model. These candidates would not have to be found in any brain during this inference stage, but should be included in the model of the white matter used to design further pattern recognition systems. What we have in mind is a model of the bundles used to label the fascicles of any new brain in a way similar to what has been developped to deal with cortical sulci [17].

Similarity measure for bundles. In order to match bundles across subjects, a shapebased similarity measure is required. Relying on iconic representations of the bundles allows the definition of discriminant measures stemming from the spatial normalization

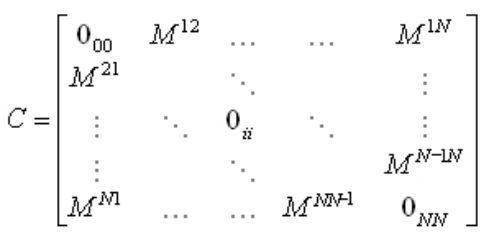

Fig. 2. The block-based structure of the matrix of similarity measures between bundles. Each $M^{i j}$ block corresponds to the one to one correlation coefficients computed between the bundles of the subject $i$ and the bundles of the subjects $j$. The $O_{i i}$ blocks would be zero-based without smoothing of the binary representations. 
towards Talairach space. For this purpose, each fascicle bundle is converted first into a binary representation lying into Talairach space. Then, to reduce the influence of the non perfect affine spatial normalization, these binary representations are convoluted with a Gaussian kernel with $3 \mathrm{~mm}$ standard deviation. Finally, for each pair of bundles $(i, j)$, the similarity $C_{i j}$ is computed as the correlation coefficient between their respective smoothed representations. With a subject-based ordering of the bundles, the similarity matrix $C$ has a block structure (see Fig. 2 and 3).

Clustering. The second stage of clustering is also performed with a K-means algorithm. Each bundle is represented by a row of the similarity matrix $C$. It should be noted that
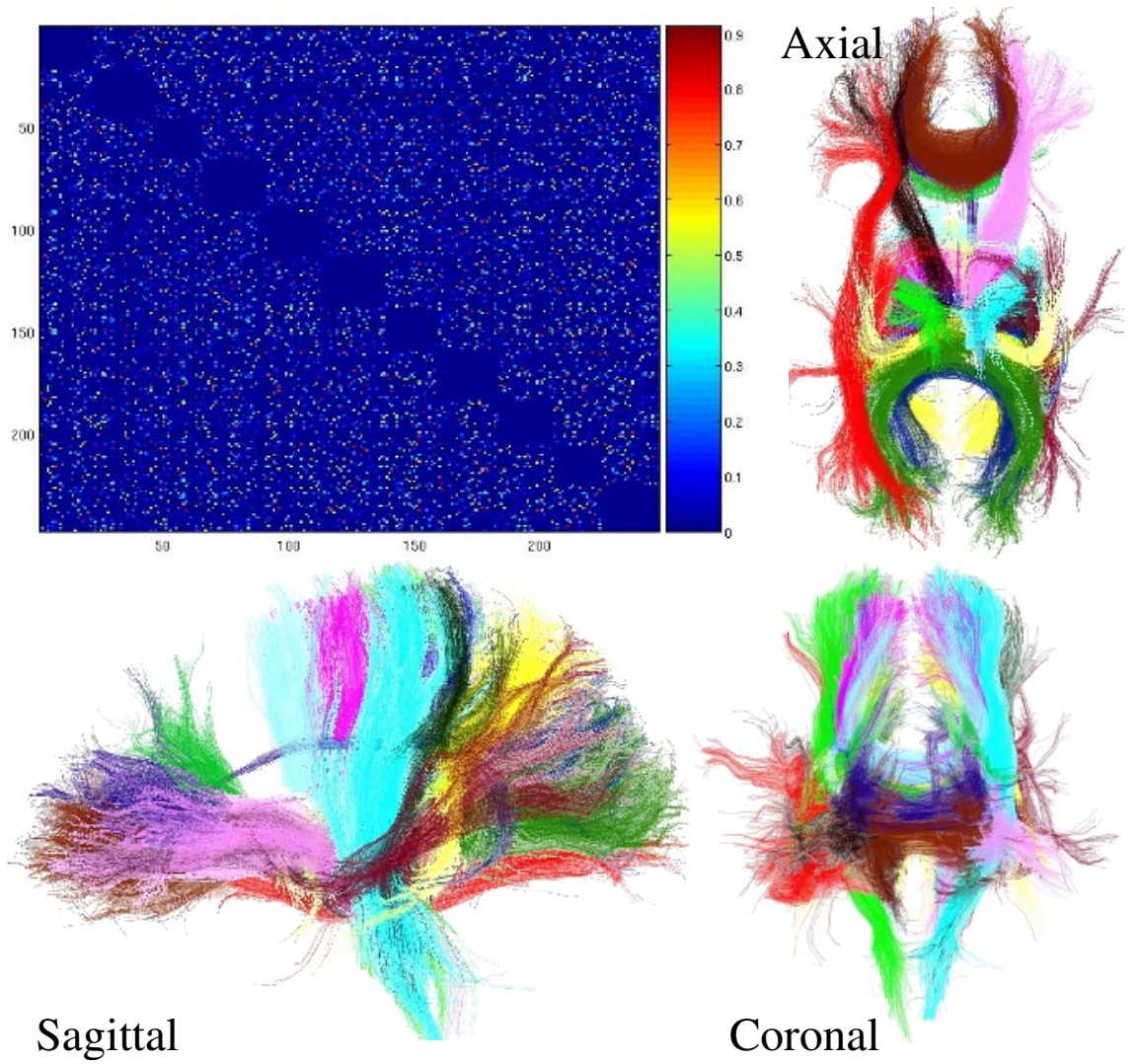

Fig. 3. Clustering the bundles across subjects: The matrix of correlation coefficient computed for all pairs of bundles and three orthogonal views of the result of the clustering. All subjects bundles have been gathered into Talairach space. Each color denotes a cluster of bundles that may correspond to a generic anatomical entity. Garbage clusters have been filtered out. Among the clusters that are candidates to stand for some known anatomical pathways, we noticed the following: (black) ALIC: Anterior Limb of Internal Capsule; (pink) ATR: Anterior Thalamic Radiation; (red) ILF: Inferior Fronto-Occipital; (red) IFO: Inferior Longitudinal Fasciculus; (braun and dark blue) GCC: Genu of Corpus Callosum; (dark green and violet blue) SCC: Splenium of corpus callosum; (light yellow) FX: Fornix. 
the K-means can merge several bundles of the same subject into the same cluster. It should be noted also that a cluster does not have to include a bundle of each subject. It should be noted finally that the K-means has to assign each bundle to a cluster. These remarks highlight the fact that a postprocessing is required to clean up the clustering before building the bundle models. The idea is first to get rid of the clusters that are not reasonable representation of a bundle, second to get rid of the bundles that look like outliers in the cluster they belong to.

Let $\mathrm{N}$ be the number of subjects. For the results presented in the following, the clusters gathering less than $N / 2$ bundles were discarded because of a lack of reproductibility across subjects. The clusters made up of more than $2 * N$ bundles were discarded as garbage collector clusters. Finally, inside each clusters, the statistics of the distance to the cluster center were computed. The outlier bundles were defined as those beyond one mean plus one standard deviation. The remaining clusters are considered as candidates to represent one of the building block of the human brain white matter organization.

\section{Results}

\subsection{Clustering the Fascicles}

Eleven subjects were processed. Their diffusion data have been acquired on a $1.5 \mathrm{~T}$ with 41 different directions for the diffusion-weighting gradient. The resulting 3D volumes have a $128 \times 128 \times 60$ resolution with $1.875 \times 1.875 \times 2$ voxel sizes. BrainVISA software was triggered with $42 \times 42 \times 205 \mathrm{~mm}$ cubic ROIs. The K-means was applied with 20 classes for each subject. After the connectivity-based postprocessing, we obtained between 20 and 25 bundles for each subject. An example of result can be found in figure 1.

\subsection{Clustering the Bundles Across a Population}

The second clustering was applied on the eleven subjects with a 25 classes K-means. After cleaning up the result, we obtained 17 reasonable clusters. The result can be visualized in figures 3 and 4 Some of these clusters seem to fit some well known bundles described in anatomical atlases, while the various bundles resulting from the split of the corpus callosum may have less anatomical meaning.

\section{Discussion}

The inference of a model of the white matter organization will become mandatory to fully exploit the information embedded into MR diffusion-weighted images. While a straightforward approach for this purpose lies in the iconic atlas and spatial normalization principles, this paper advocates an alternative which aims at infering a higher level model of the bundle organization. The work proposed in this paper is still very preliminary. However, it provides an overview of the various difficulties that have to be addressed to tackle the inference of such a model. A lot of the ad hoc choices presented above could be questioned and more sophisticated methods should be derived in the future. 


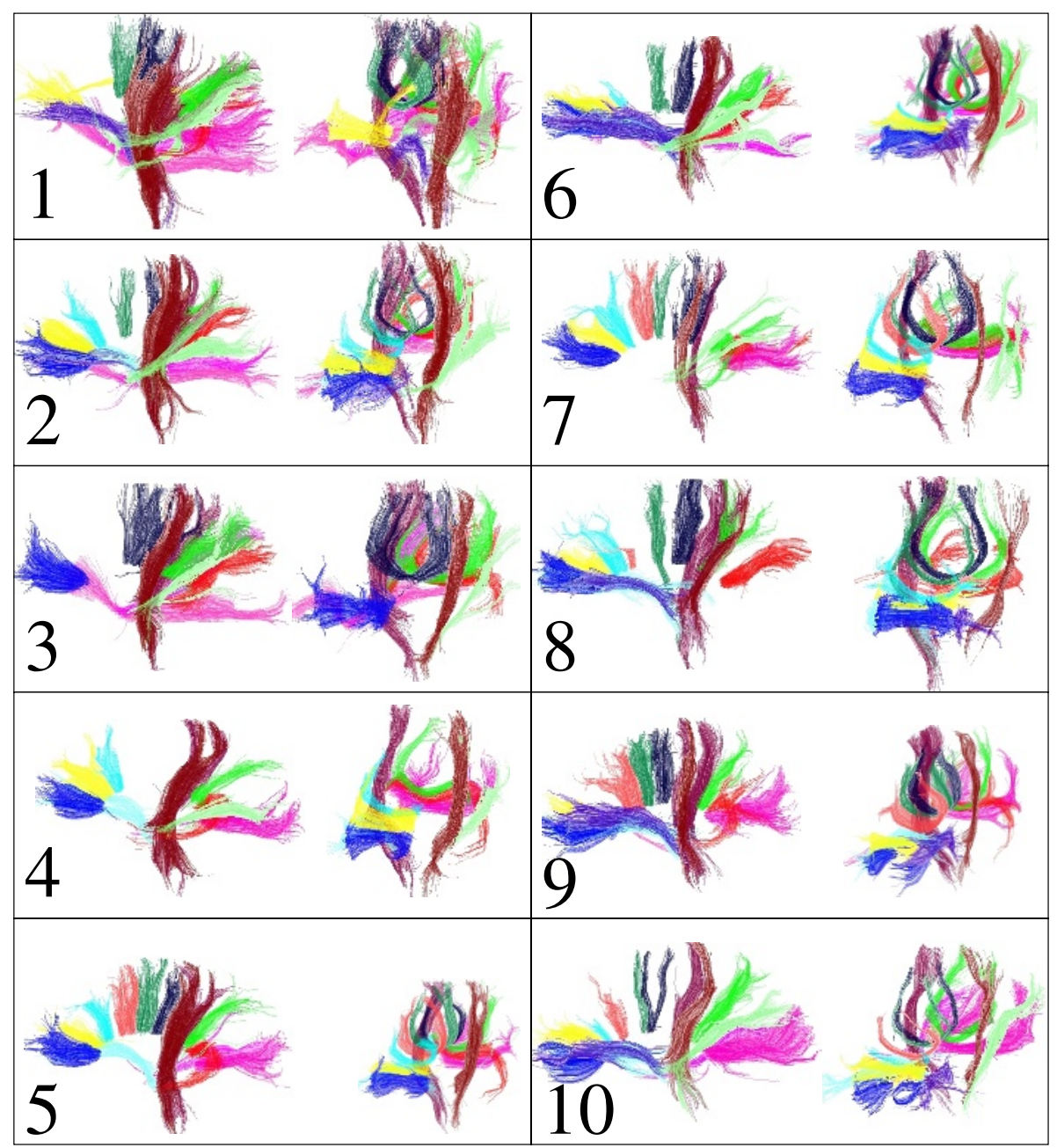

Fig. 4. Clustering the bundles across subjects: The result of the clustering for ten of the eleven subjects. For each subject two views are presented. Each color denotes a cluster that gathers bundle across most of the subjects.

The key idea proposed in our preliminary work is the two stage inference strategy: building first a bundle-based representation before trying to match bundles across subjects. This idea allows the matching to deal with reasonable data sizes. The first clustering, however, can not be questioned during the matching stage which is problematic. Therefore, future approaches may lead to build multiscale representations of the individual data. For instance, each individual bundle may be split further into smaller bundles using one more non supervised clustering. Some other multiscale representations could stem from selecting other ranges of fascicle length in order to deal with short pathways like U-fiber bundles. 
As mentioned above, the model inference does not have to find each anatomical entity in every brain. This could be the job of a dedicated pattern recognition system developed to process large databases of diffusion-weighted data. Such a system would face a simpler situation where brains are processed one by one. Hence, the matching with the bundle model could be performed at the fascicle level. Each fascicle would have to be labelled with the name of one of the entities of the model. Such a system would open the door to massive morphometric analysis of the white matter bundles, which would lead to numerous applications in neurosciences.

\section{References}

1. P. J. Basser, S. Pajevic, C. Pierpaoli, J. Duda, and A. Aldroubi. In vivo fiber tractography using DT-MRI data. MRM, 44(4):625-32, 2000.

2. T.E.J Behrens, M.W. Woolrich, M. Jenkinson, H. Johansen-Berg, R.G. Nunes, S. Clare, P.M. Matthews, J.M. Brady, and S.M. Smith. Characterization and propagation of uncertainty in diffusion-weighted MR imaging. Magnetic Resonance in Medicine, 50:1077-1088, 2003.

3. M. Björnemo, A. Brun, R. Kikinis, and C.-F. Westin. Regularized stochastic white matter tractography using diffusion tensor MRI. In MICCAI'02, LNCS 2488, pages 435-442, 2002.

4. A. Brun, H. Knutsson, H.-J. Park, M. E. Shenton, and C.-F. Westin. Clustering fiber traces using normalized cuts. In MICCAI 2004, LNCS 3216, pages 368-375, 2004.

5. T. E. Conturo, N. F. Lori, T. S. Cull, E. Akbudak, A. Z. Snyder, J. S. Shimony, R. C. McKinstry, H. Burton, and M. E. Raichle. Tracking neuronal fiber pathways in the living human brain. Proc. Natl. Acad. Sci. USA, 96:10422-10427, August 1999.

6. I. Corouge, S. Gouttard, and G. Gerig. A statistical shape model of individual fiber tracts extracted from diffusion tensor MRI. In MICCAI 2004, LNCS 3217, pages 671-679, 2004.

7. J. Dejerine. Anatomie des centres nerveux. Paris: Rueff, 1901.

8. P. Fillard, J. Gilmore, W. Lin, and G. Gerig. Quantitative analysis of white matter fiber properties along geodesic paths. In MICCAI 03, LNCS 2879, pages 16-23, 2003.

9. M. Jackowski, C. Y. Kao, R. T. Constable, and L. H. Staib. Estimation of anatomical connectivity by anisotropic front propagation and diffusion tensor imaging. In MICCAI04, LNCS 3217, pages 663-670, 2004.

10. D. K. Jones, L. D. Griffin, D. C. Alexander, M. Catani, M. A. Horsfield, R. Howard, and S. C. Williams. Spatial normalization and averaging of diffusion tensor mri data sets. Neuroimage, 17(2):592-617, 2002.

11. D Le Bihan, J.-F. Mangin, C. Poupon, C. A. Clark, S. Pappata, N. Molko N, and H. Chabriat. Diffusion tensor imaging: Concepts and applications. Journal of Magnetic Resonance Imaging, 13(4):536-546, 2001.

12. C. Lenglet, R. Deriche, and O. Faugeras. Inferring white matter geometry from diffusion tensor mri: Application to connectivity mapping. In 8th ECCV, 2004.

13. E. Ludwig and L. Klinger. Atlas cerebri humani. Basel: Karger, 1956.

14. S. Mori, B.J. Crain, V. P. Chacko, and P. C. M. van Zijl. Three dimensional tracking of axonal projections in the brain by magnetic resonance imaging. Ann. Neurol., 45:265-269, 1999.

15. G. J. M Parker and D. C. Alexander. Probabilistic Monte Carlo based mapping of cerebral connections utilising whole-brain crossing fibre information. In IPMI, pages 684-95, 2003. 
16. C. Poupon, C.A. Clark, V. Frouin, J. Régis, I. Bloch, D. Le Bihan, and J-F. Mangin. Regularization of diffusion-based direction maps for the tracking of brain white matter fascicles. NeuroImage, 12(2):184-195, 2000.

17. D. Rivière, J.-F. Mangin, D. Papadopoulos, J.-M. Martinez, V. Frouin, and J. Régis. Automatic recognition of cortical sulci of the human brain using a congregation of neural networks. Medical Image Analysis, 6(2):77-92, 2002.

18. D. S. Tuch. Diffusion MRI of complex tissue structure. PhD thesis, MIT, 2002.

19. D. Weinstein, G. Kindlmann, and E. Lundberg. Tensorlines: Advection-diffusion based propagation through diffusion tensor fields. In IEEE Visualization, pages 249-254, 1999.

20. D. Xu, S. Mori, D. Shen, P. C. van Zijl, and C. Davatzikos. Spatial normalization of diffusion tensor fields. Magn Reson Med, 50(1):175-182, 2003. 